\title{
GENERALIZED CAHN-HILLIARD EQUATIONS BASED ON A MICROFORCE BALANCE
}

\section{ALAIN MIRANVILLE}

Received 30 April 2002 and in revised form 23 September 2002

We present some models of Cahn-Hilliard equations based on a microforce balance proposed by M. Gurtin. We then study the existence and uniqueness of solutions.

\section{Introduction}

The Cahn-Hilliard equation is very important in materials science. It is a conservation law which describes important qualitative behaviors of two-phase systems, namely, the phase separation process in a binary alloy. This phenomenon can be observed, for instance, when the alloy is cooled down sufficiently. One then observes a partial nucleation (i.e., the apparition of nucleides in the material) or a total nucleation (the socalled spinodal decomposition: the mixture quickly becomes inhomogeneous, forming a fine-grained structure). We refer the reader to [15] for more details; see also $[54,55]$ for a qualitative study of the spinodal decomposition.

The starting point of the Cahn-Hilliard theory is a free energy $\psi=$ $\psi(\rho, \nabla \rho)$ of the form

$$
\psi(\rho, \nabla \rho)=f(\rho)+\frac{\alpha}{2}|\nabla \rho|^{2}
$$

$\alpha>0$ (it is related to the surface tension), also called Ginzburg-Landau free energy, where $\rho$ is the order parameter (corresponding to the density of the atoms) and $f$ is a double-well potential whose wells correspond to the phases of the material. Usually, one considers polynomials of degree four; more generally, one can consider polynomials with arbitrary even 
degree. Logarithmic potentials are also very important; actually, polynomial potentials can be seen as approximations of logarithmic potentials in certain situations. Such a form of free energy is obtained, at first approximation, by assuming that the local free energy per molecule in a region of nonuniform composition depends both on the local composition and on that of the immediate environment. We refer the reader to [16] for more details. Then, one considers the mass balance

$$
\frac{\partial \rho}{\partial t}=-\operatorname{div} h
$$

where $h$ is the mass flux, which is related to the chemical potential $\mu$ by the following (postulated; see [25]) constitutive equation:

$$
h=-\kappa \nabla \mu,
$$

where $\kappa$ is the mobility. We assume here that it is a strictly positive constant; more generally, $\kappa$ will depend on $\rho$. Now, the standard definition of $\mu$ is that it is the derivative of the free energy with respect to $\rho$. Here, this is incompatible with the presence of $\nabla \rho$ in the free energy, so that the definition has to be adapted. So, instead, $\mu$ is defined as a variational derivative of $\psi$ and we find that

$$
\mu=-\alpha \Delta \rho+f^{\prime}(\rho)
$$

This relation is nevertheless well accepted and physically realistic (from a thermodynamical point of view). We note however that it is derived by assuming that the system is not far from equilibrium. Finally, we deduce from (1.2), (1.3), and (1.4) the so-called Cahn-Hilliard equation

$$
\frac{\partial \rho}{\partial t}+\alpha \kappa \Delta^{2} \rho-\kappa \Delta f^{\prime}(\rho)=0
$$

This equation has been extensively studied; see, in particular, [30, 65] for reviews on the Cahn-Hilliard equation. Existence and uniqueness results are obtained in [35, 61] for polynomial potentials and in [34] for logarithmic potentials (see also [33] for nonconstant mobilities and logarithmic potentials with degenerating mobilities and [26] for logarithmic potentials with constant mobilities); we also refer to [63] for the use of energy methods and to [13] for an existence result in the whole space. The first result on the existence of finite-dimensional attractors (and also inertial manifolds) is obtained in [62] (see also [68]) for a polynomial potential of degree four at most in three space dimensions. This restriction is relaxed in [51] (see also [20]) where further regularity results are obtained. The existence of finite-dimensional attractors for 
logarithmic potentials is proved in [26]. Finally, we refer the reader to $[3,4,5,23,31,32,38]$ for the approximation of the Cahn-Hilliard equation by finite elements, to [37] for finite differences, and to [22] for spectral methods.

Now, Gurtin notes in [47] that the classical derivation is physically sound and important, but makes several objections as follows:

(1) it requires a priori specifications of the constitutive equations (we recall that (1.3) is postulated);

(2) it is not clear how it can be generalized in the presence of processes such as deformation or heat transfer;

(3) there is no clear separation of balance laws from constitutive equations (such a separation is one of the major advances in nonlinear continuum mechanics over the past thirty years). Also, it is not clear whether or not there is an underlying balance law that can form a basis for more complete theories.

So, what distinguishes the approach of Gurtin from other macroscopic theories of order parameters is

(1) the separation of balance laws from constitutive equations;

(2) the introduction of a new balance law for microforces (we can note that microforces describe forces associated with microscopic configurations of atoms, whereas standard forces are associated with macroscopic length scales, which can explain the need for a separate balance law for microforces).

This second point is further motivated by the belief that fundamental physical laws involving energy should account for the working associated with each operative kinematical process (that is associated with the order parameter in the Cahn-Hilliard theory). Consequently, it seems plausible that there should be microforces whose working accompanies changes in the order parameter. Fried and Gurtin describe this working through terms of the form $\partial \rho / \partial t$ so that the microforces are scalar rather than vector quantities; see [39,40]. More precisely, the microforce system is characterized by the following physical quantities: the microstress $\zeta$ (a vector) and the microforce $\pi$ (a scalar). These quantities are related by the (local) microforce balance

$$
\operatorname{div} \zeta+\pi=\gamma,
$$

where $\gamma=\gamma(x, t)$ corresponds to external microforces.

Finally, the two basic balance laws for the generalized Cahn-Hilliard theory are the mass balance

$$
\frac{\partial \rho}{\partial t}=-\operatorname{div} h+m,
$$


where $m=m(x, t)$ is an external mass supply, and the microforce balance (1.6). Thus there remains to obtain constitutive equations; these will be derived by considering the restrictions imposed by the second law of thermodynamics (see [47] and Section 2 below). Actually, Gurtin considers a purely mechanical version of the second law, which states that the rate at which the free energy increases cannot exceed the sum of the working and of the energy inflow due to mass transport.

At this stage, we should also mention a very interesting generalization of the Cahn-Hilliard equation proposed by Giacomin and Lebowitz in [45] (see also [46]). These authors make the following objection to the classical Cahn-Hilliard theory: it does not seem to arise from an exact macroscopic description of microscopic models of interacting particles (we can note however that the Cahn-Hilliard equation can be derived from certain mesoscopic Ginzburg-Landau continuous-spin models, see [6]). Based on stochastic arguments, Giacomin and Lebowitz derive rigorously, by considering a lattice gas with long range Kac potentials (i.e., the interaction energy between two particles at $x$ and $y\left(x, y \in \mathbb{Z}^{n}, n\right.$ being the space dimension) is given by $\gamma^{n} \mathcal{K}(\gamma|x-y|), \gamma>0$ being sent to 0 and $\mathcal{K}$ being a smooth function), a Cahn-Hilliard type equation with a (total) nonlocal free energy of the form

$$
\Psi(\rho)=\int_{T^{n}}\left[f(\rho(x))+\rho(x) \int_{T^{n}} \mathcal{K}(|x-y|)(1-\rho(y)) d y\right] d x,
$$

where $T^{n}$ is the $n$-dimensional torus. We can note that, in that case, the definition of $\mu$ is the standard one. Furthermore, rewriting the total free energy in the form

$$
\begin{aligned}
\Psi(\rho)=\int_{T^{n}}\left[f(\rho(x))+\kappa_{1}(x) \rho(x)(1-\rho(x))\right. \\
\left.\quad+\frac{1}{2} \int_{T^{n}} \mathcal{K}(|x-y|)|\rho(x)-\rho(y)|^{2} d y\right] d x,
\end{aligned}
$$

$\mathcal{\kappa}_{1}(x)=\int_{T^{n}} \mathcal{K}(|x-y|) d y$, we can, by expanding the last term and keeping only some terms of the expansion, recover the Ginzburg-Landau free energy (this will be reasonable if the scale on which the free energy varies is large compared to $\gamma^{-1}$; the macroscopic evolution is observed here on the spatial scale $\gamma^{-1}$ and the time scale $\left.\gamma^{-2}\right)$. We refer the reader to $[45,46]$ for more details; see also [41, 42] for the mathematical study of such models.

Finally, we briefly mention some other interesting generalizations of the Cahn-Hilliard equation: multicomponent alloys and mechanical effects (see, e.g., $[7,8,36,43,44]$ ), nonisothermal phase separation (see $[1,2,41,67])$, the stochastic Cahn-Hilliard equation (see [24, 29]), and the viscous Cahn-Hilliard equation (see, e.g., [64]). We can note that the 
viscous Cahn-Hilliard equation is obtained as a singular limit of a phasefield model for phase transitions; actually, the Cahn-Hilliard equation can also be obtained as a singular limit of a phase-field model (see, e.g., [14]). We can finally mention models in which the Cahn-Hilliard equation is coupled with the Allen-Cahn equation (see, e.g., $[17,66]$ ) or the Navier-Stokes equations (see [11, 12, 21, 48, 49, 50, 52, 53]).

\section{Setting of the problem}

In the classical Cahn-Hilliard theory, the independent constitutive variables are $\rho$ and $\nabla \rho$. Then, as mentioned above, $\mu$ is given, constitutively, as a function of $\rho$ and $\nabla \rho$, assuming that the system is close to equilibrium. Since we want to consider systems that are sufficiently far from equilibrium, it is reasonable to add $\mu$ and $\nabla \mu$ to the list of independent constitutive variables. We will also add the kinetics, that is, $\partial \rho / \partial t$ to the list of constitutive variables (we recall that the working of the internal microforces is modelled through terms of this form).

So, we set here $Z=(\rho, \nabla \rho, \partial \rho / \partial t, \mu, \nabla \mu)$ and we assume that $\psi, \zeta$, and $\pi$ depend on $Z$. In order to derive the equations, we have the balance laws (1.6) and (1.7). Then, the second law of thermodynamics yields the dissipation inequality (see [47] for more details)

$$
\begin{aligned}
{\left[\partial_{\rho} \psi(Z)+\pi(Z)-\right.} & \mu] \frac{\partial \rho}{\partial t}+\left[\partial_{\nabla \rho} \psi(Z)-\zeta(Z)\right] \cdot \nabla \frac{\partial \rho}{\partial t}+\left[\partial_{\partial \rho / \partial t} \psi(Z)\right] \frac{\partial^{2} \rho}{\partial t^{2}} \\
+ & {\left[\partial_{\mu} \psi(Z)\right] \frac{\partial \mu}{\partial t}+\left[\partial_{\nabla \mu} \psi(Z)\right] \cdot \nabla \frac{\partial \mu}{\partial t}+h(Z) \cdot \nabla \mu \leq 0 }
\end{aligned}
$$

for every fields $\rho$ and $\mu$, which yields that

$$
\psi=\psi(\rho, \nabla \rho),
$$

as expected, and

$$
\zeta=\partial_{\nabla \rho} \psi
$$

together with the dissipation inequality

$$
\left[\partial_{\rho} \psi(\rho, \nabla \rho)+\pi(Z)-\mu\right] \frac{\partial \rho}{\partial t}+h(Z) \cdot \nabla \mu \leq 0,
$$

for every fields $\rho$ and $\mu$. A consequence of (2.4) is the existence of constitutive moduli $\beta=\beta(Z)$ (a scalar; it characterizes the action of the internal 
microforces), $a=a(Z), b=b(Z)$ (two vectors; the vector $a$ also characterizes the action of the internal microforces, whereas $b$ characterizes the anisotropy of the material (it vanishes for isotropic materials)), and the mobility tensor $B=B(Z)$ (a positive semidefinite matrix) such that

$$
\begin{gathered}
h=-a \frac{\partial \rho}{\partial t}-B \nabla \mu, \\
\partial_{\rho} \psi+\pi-\mu=-\beta \frac{\partial \rho}{\partial t}-b \cdot \nabla \mu,
\end{gathered}
$$

and (2.4) is satisfied.

For simplicity, we assume from now on that the constitutive moduli $\beta, a, b$, and $B$ are constant (this can be assumed at first approximation, see [47, Appendix B]). Taking then the classical Ginzburg-Landau free energy (1.1), we deduce from (1.6), (1.7), and (2.5) the following system of equations for $\rho$ and $\mu$ :

$$
\begin{gathered}
\frac{\partial \rho}{\partial t}-a \cdot \nabla \frac{\partial \rho}{\partial t}=\operatorname{div}(B \nabla \mu)+m \\
\mu-b \cdot \nabla \mu=\beta \frac{\partial \rho}{\partial t}-\alpha \Delta \rho+f^{\prime}(\rho)-\gamma
\end{gathered}
$$

where (due to (2.4))

$$
\beta x^{2}+(a+b) \cdot y x+B y \cdot y \geq 0, \quad \forall x \in \mathbb{R}, \forall y \in \mathbb{R}^{n}
$$

and where denotes the usual Euclidian scalar product.

Actually, the equation that is generally called the Cahn-Hilliard equation is that obtained by eliminating the chemical potential in the above system. Before doing so, we first transform the equations.

We assume from now on that $\rho$ and $\mu$ (and also $m$ and $\gamma$ ) are periodic in space. Actually, Neumann boundary conditions are preferred in the Cahn-Hilliard theory. However, it is not clear in general what the proper conditions should be here; we refer the interested reader to $[18,56,57$, $58,59]$ for discussions on this subject and for the mathematical study of simplified models.

Integrating (2.6) over the spatial domain $\Omega=\prod_{i=1}^{n}\left(0, L_{i}\right), L_{i}>0, i=$ $1, \ldots, n$, and $n=2$ or 3 , we find that

$$
\frac{d}{d t} \int_{\Omega} \rho d x=\int_{\Omega} m d x .
$$


Thus, the average of $\rho$ is not conserved in general (contrarily to the classical Cahn-Hilliard equation). Now, this conservation property is sometimes important for the mathematical study of the problem (see, e.g., $[62,68])$. So, in order to obtain a conservation law (for the order parameter), we set

$$
q=\rho-\frac{1}{\operatorname{Vol}(\Omega)} \int_{0}^{t} d \tau \int_{\Omega} m d x .
$$

We then obtain the following system of equations:

$$
\begin{gathered}
\frac{\partial q}{\partial t}-a \cdot \nabla \frac{\partial q}{\partial t}=\operatorname{div}(B \nabla \mu)+g, \\
\mu-b \cdot \nabla \mu=\beta \frac{\partial q}{\partial t}-\alpha \Delta q+f^{\prime}(q+\varphi)+h,
\end{gathered}
$$

where

$$
\begin{aligned}
& g=g(x, t)=m-\frac{1}{\operatorname{Vol}(\Omega)} \int_{\Omega} m d x, \\
& h=h(x, t)=\frac{\beta}{\operatorname{Vol}(\Omega)} \int_{\Omega} m d x-\gamma, \\
& \varphi=\varphi(t)=\frac{1}{\operatorname{Vol}(\Omega)} \int_{0}^{t} d \tau \int_{\Omega} m d x,
\end{aligned}
$$

with which we associate the boundary conditions

$$
q \text { and } \mu \text { are } \Omega \text {-periodic, }
$$

and the initial condition

$$
q(x, 0)(=\rho(x, 0))=q_{0}(x) .
$$

Taking now the $\operatorname{div} B \nabla$ of (2.12) and injecting the value of $\operatorname{div}(B \nabla \mu)$ given by (2.11) into the equation that we obtain, we have the following generalized Cahn-Hilliard equation:

$$
\begin{gathered}
\frac{\partial q}{\partial t}-d \cdot \nabla \frac{\partial q}{\partial t}-\operatorname{div}\left(\tilde{B} \nabla \frac{\partial q}{\partial t}\right)+\alpha \operatorname{div}(B \nabla \Delta q)-\operatorname{div}\left(B \nabla f^{\prime}(q+\varphi)\right)=k \\
q \text { is } \Omega \text {-periodic, } \\
q(x, 0)=q_{0}(x)
\end{gathered}
$$


where $d=a+b, \tilde{B}=\beta B-(1 / 2)\left(a^{t} b+b^{t} a\right)$, and

$$
k=k(x, t)=g-b \cdot \nabla g+\operatorname{div}(B \nabla h) .
$$

Here, it follows from (2.8) that, when $B$ is symmetric (which will be assumed in Section 3 for the study of (2.16), (2.17), and (2.18)), the matrix $\tilde{B}$, which is then symmetric, is positive semidefinite. Furthermore, the conservation of the average of $q$ will be important for the study of (2.16), (2.17), and (2.18) (it is not essential for the study of the first formulation).

We refer the reader to $[9,10,19,27,56,57,58,60]$ for the study of some (simplified) models based on the above equations, with an emphasis on the study of finite-dimensional attractors and Neumann-type boundary conditions.

We finally assume that $m=\gamma=0$ and that $\rho$ is known (and is regular). We can then obtain the chemical potential $\mu$ by solving (2.6) and find that

$$
\mu=-(-\operatorname{div} B \nabla)^{-1}\left(\frac{\partial \rho}{\partial t}-a \cdot \nabla \frac{\partial \rho}{\partial t}\right)+\frac{1}{\operatorname{Vol}(\Omega)} \int_{\Omega} f^{\prime}(\rho) d x
$$

or by solving (2.7) (for simplicity, we assume that $n=3$ and $b_{1} \neq 0, b=$ $\left.\left(b_{1}, b_{2}, b_{3}\right)\right)$ which yields

$$
\begin{aligned}
& \mu=(I-b \cdot \nabla)^{-1}\left[-\alpha \Delta \rho+\beta \frac{\partial \rho}{\partial t}+f^{\prime}(\rho)\right] \\
&=\frac{1}{b_{1}} \int_{x_{1}}^{+\infty} e^{-s / b_{1}}\left[-\alpha \Delta \rho+\beta \frac{\partial \rho}{\partial t}+f^{\prime}(\rho)\right] \\
& \quad \times\left(s, x_{2}-\frac{b_{2}}{b_{1}} x_{1}+\frac{b_{2}}{b_{1}} s, x_{3}-\frac{b_{3}}{b_{1}} x_{1}+\frac{b_{3}}{b_{1}} s, t\right) d s .
\end{aligned}
$$

We can note that both expressions for $\mu$ are not satisfactory, say, for practical purposes.

We now set $\phi=\int_{0}^{t}(I-a \cdot \nabla)^{-1} \mu d s$. Then, the function $\phi$ is a solution of

$$
\begin{gathered}
\frac{\partial \phi}{\partial t}-d \cdot \nabla \frac{\partial \phi}{\partial t}-\operatorname{div}\left(\tilde{B} \nabla \frac{\partial \phi}{\partial t}\right)+\alpha \Delta(\operatorname{div}(B \nabla \phi)+\rho(0)) \\
-f^{\prime}(\operatorname{div}(B \nabla \phi)+\rho(0))=0, \\
\phi \text { is } \Omega \text {-periodic, } \\
\left.\phi\right|_{t=0}=0 .
\end{gathered}
$$


Actually, for an easier (mathematical) treatment of the nonlinear term, we set

$$
\theta=-(-\operatorname{div} B \nabla)^{-1}\left(\rho(0)-\frac{1}{\operatorname{Vol}(\Omega)} \int_{\Omega} \rho(0) d x\right)
$$

and we consider the following problem for $\Phi=\phi+\theta$ :

$$
\begin{gathered}
\frac{\partial \Phi}{\partial t}-d \cdot \nabla \frac{\partial \Phi}{\partial t}-\operatorname{div}\left(\tilde{B} \nabla \frac{\partial \Phi}{\partial t}\right)+\alpha \operatorname{div}(B \nabla \Delta \Phi)-g^{\prime}(\operatorname{div}(B \nabla \Phi))=0 \\
\Phi \text { is } \Omega \text {-periodic, } \\
\left.\Phi\right|_{t=0}=\theta
\end{gathered}
$$

where $g(s)=f\left(s+(1 / \operatorname{Vol}(\Omega)) \int_{\Omega} \rho(0) d x\right)$. We then have

$$
\begin{gathered}
\rho=\operatorname{div}(B \nabla \Phi)+\frac{1}{\operatorname{Vol}(\Omega)} \int_{\Omega} \rho(0) d x, \\
\mu=\frac{\partial \Phi}{\partial t}-a \cdot \nabla \frac{\partial \Phi}{\partial t}
\end{gathered}
$$

and we now note that $\mu$ (and also $\rho$ ) are given, in terms of $\Phi$, by simple explicit expressions. Also, (2.24) is very similar to the generalized CahnHilliard equation (2.16) (although we do not have a conservation law), with $k=\varphi=0$. Actually, we could have a similar result when $m$ and $\gamma$ do not vanish. Unfortunately, in that case, the new system would not be convenient in general.

Remark 2.1. We could also consider models in which the deformations of the material are taken into account (these deformations are essentially due to the displacement of atoms in the material). In that case, the gradient of the displacement is added to the list of independent constitutive variables. We refer the reader to [47] for more details; see also $[9,18,44,56,57,58,59]$ for the mathematical study of such models.

Remark 2.2. We could further generalize the models described above by also adding $\nabla(\partial \rho / \partial t)$ to the list of independent constitutive variables. This will be studied in [28]. 


\section{Existence and uniqueness of solutions}

We first consider formulations (2.11), (2.12), (2.14), and (2.15).

We set $H=L^{2}(\Omega)$ and $V=H_{\text {per }}^{1}(\Omega)$, which we endow with their usual scalar products and norms; in particular, we denote by $(\cdot, \cdot)$ the usual scalar product on $H$ and by $|\cdot|$ the associated norm.

We then associate with (2.11), (2.12), (2.14), and (2.15) the following variational formulation, for $T>0$ given.

Find $(q, \mu):[0, T] \rightarrow V$ such that

$$
\begin{aligned}
\frac{d}{d t}[(q, r)+(q, a \cdot \nabla r)]= & -(B \nabla \mu, \nabla r)+(g, r), \quad \forall r \in V, \\
(\mu, r)+(\mu, b \cdot \nabla r)= & \alpha(\nabla q, \nabla r)+\beta \frac{d}{d t}(q, r) \\
& +\left(f^{\prime}(q+\varphi), r\right)+(h, r), \quad \forall r \in V, \\
q(0)= & q_{0} .
\end{aligned}
$$

We assume that the constitutive moduli satisfy the following coercivity assumption, stronger than (2.8):

$$
\beta x^{2}+(a+b) \cdot y x+B y \cdot y \geq c\left(x^{2}+|y|^{2}\right), \quad \forall x \in \mathbb{R}, \forall y \in \mathbb{R}^{n}
$$

In particular, (3.2) yields that $\beta>0$ and that the mobility tensor $B$ is positive definite.

We then make the following assumptions on the nonlinear term:

$$
\begin{gathered}
f \text { is of class } \mathcal{C}^{1}, \\
c_{1} s^{2 p+2}-c_{2} \leq f(s) \leq c_{3} s^{2 p+2}+c_{4}, \quad \forall s \in \mathbb{R}, c_{1}, c_{3}>0, c_{2}, c_{4} \geq 0, \\
\left|f^{\prime}(s)\right| \leq c_{5}|s|^{2 p+1}+c_{6}, \quad \forall s \in \mathbb{R}, c_{5}, c_{6} \geq 0,
\end{gathered}
$$

where $p \geq 1$ if $n=2$ and $p \in[1,2]$ if $n=3$. For instance, polynomials of degree $2 p+2$ with strictly positive leading coefficient satisfy (3.3), (3.4), and (3.5). We note that logarithmic potentials do not satisfy these conditions; actually, for such potentials, we are able to study very simplified models only (see $[9,19]$ ).

Finally, we make the following assumptions on $g, h$, and $\varphi$ :

$$
\begin{gathered}
g, h \in L_{\mathrm{loc}}^{\infty}\left(\mathbb{R}^{+} ; L^{\infty}(\Omega)\right), \\
\varphi \in \mathcal{C}^{1}\left(\mathbb{R}^{+}\right) .
\end{gathered}
$$

We have the following result. 
Theorem 3.1. Assume that (3.2), (3.3), (3.4), (3.5), and (3.6) hold. Then, for every $q_{0} \in V$, (3.1) possesses at least one solution $(q, \mu)$ such that $q \in$ $\mathcal{C}([0, T] ; H) \cap L^{\infty}(0, T ; V), \partial q / \partial t \in L^{2}(0, T ; H)$, and $\mu \in L^{2}(0, T ; V)$, for all $T>0$. If , furthermore, $f \in \mathcal{C}^{2}(\mathbb{R})$ and $\left|f^{\prime \prime}(s)\right| \leq c_{7}|s|^{2 p}+c_{8}$, for all $s \in \mathbb{R}$, $c_{7}, c_{8} \geq 0$, with $p=1$ if $n=3$, then this solution is unique.

Proof. Let $A$ denote the operator $-\Delta$ with domain $H_{\text {per }}^{2}(\Omega)$. We call $0=$ $\lambda_{1}<\lambda_{2} \leq \cdots \leq \lambda_{m}$ the first $m$ eigenvalues of $A$ and $\varphi_{1}, \ldots, \varphi_{m}$ the associated eigenvectors. We further assume that the $\varphi_{j}$ are orthonormal in $H$. We then consider the approximate problem

Find $q^{m}$ and $\mu^{m}$ such that

$$
\begin{gathered}
q^{m}=\sum_{i=1}^{m} c_{i}^{m}(t) \varphi_{i}(x), \quad \mu^{m}=\sum_{i=1}^{m} d_{i}^{m}(t) \varphi_{i}(x), \\
\frac{d}{d t}\left[\left(q^{m}, \varphi_{j}\right)+\left(q^{m}, a \cdot \nabla \varphi_{j}\right)\right]= \\
+\left(B \nabla \mu^{m}, \nabla \varphi_{j}\right) \\
+\left(g, \varphi_{j}\right), \quad j=1, \ldots, m, \\
\left(\mu^{m}, \varphi_{j}\right)+\left(\mu^{m}, b \cdot \nabla \varphi_{j}\right)=\alpha\left(\nabla q^{m}, \nabla \varphi_{j}\right)+\beta \frac{d}{d t}\left(q^{m}, \varphi_{j}\right) \\
+\left(f^{\prime}\left(q^{m}+\varphi\right), \varphi_{j}\right)+\left(h, \varphi_{j}\right), \quad j=1, \ldots, m, \\
q^{m}(0)=q_{0}^{m},
\end{gathered}
$$

where $q_{0}^{m}$ is the orthogonal projection in $H$ of $q_{0}$ onto $\operatorname{Span}\left(\varphi_{1}, \ldots, \varphi_{m}\right)$. This system can be rewritten, omitting for simplicity the superscript $m$ in $c_{i}^{m}$ and $d_{i}^{m}$,

$$
\begin{gathered}
c_{i}^{\prime}+\sum_{j=1}^{m} c_{j}^{\prime}\left(\varphi_{j}, a \cdot \nabla \varphi_{i}\right)=-\sum_{j=1}^{m} d_{j}\left(B \nabla \varphi_{j}, \nabla \varphi_{i}\right)+\left(g, \varphi_{i}\right), \\
d_{i}+\sum_{j=1}^{m} d_{j}\left(\varphi_{j}, b \cdot \nabla \varphi_{i}\right)=\alpha \lambda_{i} c_{i}+\beta c_{i}^{\prime}+\left(f^{\prime}\left(q^{m}+\varphi\right), \varphi_{i}\right)+\left(h, \varphi_{i}\right),
\end{gathered}
$$

where $i=1, \ldots, m$.

We note that $\varphi_{1}=$ Const. Thus, since $\int_{\Omega} g d x=0$, (3.11) yields, for $i=1$,

$$
c_{1}=\text { Const }
$$

this constant depending only on $q_{0}^{m}$. This will give the conservation of the average of $q$ when passing to the limit. 
We set

$$
C=\left(\begin{array}{c}
c_{1} \\
\vdots \\
c_{m}
\end{array}\right), \quad D=\left(\begin{array}{c}
d_{1} \\
\vdots \\
d_{m}
\end{array}\right), \quad M=\left(\begin{array}{cc}
M_{1} & M_{2} \\
M_{3} & -\beta I
\end{array}\right)
$$

where $I$ is the identity matrix, $\left(M_{1}\right)_{i j}=-\left(B \nabla \varphi_{i}, \nabla \varphi_{j}\right),\left(M_{2}\right)_{i j}=-\delta_{i j}-$ $\left(a \cdot \nabla \varphi_{i}, \varphi_{j}\right),\left(M_{3}\right)_{i j}=\delta_{i j}-\left(\varphi_{i}, b \cdot \nabla \varphi_{j}\right), i, j=1, \ldots, m$, and

$$
F(t, C)=\left(\begin{array}{c}
-\left(g, \varphi_{i}\right)_{i=1, \ldots, m} \\
\left(\left(h, \varphi_{i}\right)+\alpha \lambda_{i} c_{i}+\left(f^{\prime}\left(q^{m}+\varphi\right), \varphi_{i}\right)\right)_{i=1, \ldots, m}
\end{array}\right) .
$$

Then, (3.11) and (3.12) is equivalent to

$$
M\left(\begin{array}{l}
D \\
C^{\prime}
\end{array}\right)=F(t, C)
$$

We set $\tilde{M}=\left(M_{i j}\right)_{i, j=2, \ldots, 2 m}\left(M=\left(M_{i j}\right)_{i, j=1, \ldots, 2 m}\right)$. Our aim is first to prove that $\tilde{M}$ is invertible. Let

$$
X=\left(\begin{array}{c}
x_{1} \\
\tilde{X}
\end{array}\right), \quad \tilde{X}=\left(\begin{array}{c}
x_{2} \\
\vdots \\
x_{m}
\end{array}\right), \quad Y=\left(\begin{array}{c}
y_{1} \\
\vdots \\
y_{m}
\end{array}\right)
$$

belong to $R^{m}$. We set $\bar{X}=\sum_{i=1}^{m} x_{i} \varphi_{i}$ and $\bar{Y}=\sum_{i=1}^{m} y_{i} \varphi_{i}$. Noting that $\varphi_{1}=$ Const, we have

$$
\left(\tilde{M}\left(\begin{array}{l}
\tilde{X} \\
Y
\end{array}\right),\left(\begin{array}{l}
\tilde{X} \\
Y
\end{array}\right)\right)=-\beta|\bar{Y}|^{2}-((a+b) \cdot \nabla \bar{X}, \bar{Y})-(B \nabla \bar{X}, \nabla \bar{X}) .
$$

Therefore, thanks to (3.2), this quantity is negative and vanishes if and only if $\bar{X}=$ Const and $\bar{Y}=0$, which implies that $\tilde{M}$ is invertible.

We now note that $M_{1 j}=-\delta_{(m+1) j}$ and $M_{i 1}=\delta_{i(m+1)}$. Therefore, we can solve (3.16) (we note that $M$ is not invertible) and obtain the existence of a local (in time) solution for (3.8), (3.9), and (3.10).

We then set $\varepsilon_{m}(t)=(\alpha / 2)\left|\nabla q^{m}\right|^{2}+\int_{\Omega} f\left(q^{m}+\varphi\right) d x$. We have

$$
\begin{array}{r}
\frac{d \varepsilon_{m}}{d t}=-\beta\left|\frac{\partial q^{m}}{\partial t}\right|^{2}-\left((a+b) \cdot \nabla \mu^{m}, \frac{\partial q^{m}}{\partial t}\right)-\left(B \nabla \mu^{m}, \nabla \mu^{m}\right) \\
+\left(f^{\prime}\left(q^{m}+\varphi\right), \frac{\partial \varphi}{\partial t}\right)-\left(h, \frac{\partial q^{m}}{\partial t}\right)+\left(g, \mu^{m}\right)
\end{array}
$$


which yields, thanks to (3.2),

$$
\frac{d \varepsilon_{m}}{d t}+c\left(\left|\nabla \mu^{m}\right|^{2}+\left|\frac{\partial q^{m}}{\partial t}\right|^{2}\right) \leq\left(f^{\prime}\left(q^{m}+\varphi\right), \frac{\partial \varphi}{\partial t}\right)-\left(h, \frac{\partial q^{m}}{\partial t}\right)+\left(g, \mu^{m}\right) .
$$

Taking $j=1$ in (3.9), we have

$$
\int_{\Omega} \mu^{m} d x=\int_{\Omega} f^{\prime}\left(q^{m}+\varphi\right) d x+\int_{\Omega} h d x
$$

which yields, thanks to (3.4) and (3.5),

$$
\left|\int_{\Omega} \mu^{m} d x\right| \leq c \int_{\Omega} f\left(q^{m}+\varphi\right) d x+c^{\prime}\left|\nabla \mu^{m}\right|+c^{\prime \prime}(T)
$$

where $c$ is strictly positive. It then follows from (3.20) and (3.22) that

$$
\frac{d \varepsilon_{m}}{d t}+c\left(\left|\nabla \mu^{m}\right|^{2}+\left|\frac{\partial q^{m}}{\partial t}\right|^{2}\right) \leq c^{\prime}(T) \varepsilon_{m}+c^{\prime \prime}(T)
$$

where $c$ and $c^{\prime}(T)$ are strictly positive. We finally deduce from (3.22) and (3.23), using (3.4), that the solution is global and we obtain the necessary a priori estimates, which allows us to pass to the limit.

Now let $\left(q_{1}, \mu_{1}\right)$ and $\left(q_{2}, \mu_{2}\right)$ be two solutions of (3.1). We set $q=q_{1}-q_{2}$ and $\mu=\mu_{1}-\mu_{2}$. We then have

$$
\begin{aligned}
\frac{d}{d t}[(q, r)+(q, a \cdot \nabla r)]= & -(B \nabla \mu, \nabla r), \quad \forall r \in V, \\
(\mu, r)+(\mu, b \cdot \nabla r)= & \alpha(\nabla q, \nabla r)+\beta \frac{d}{d t}(q, r) \\
& +\left(f^{\prime}\left(q_{1}+\varphi\right)-f^{\prime}\left(q_{2}+\varphi\right), r\right), \quad \forall r \in V, \\
q(0)= & 0 .
\end{aligned}
$$

We take $r=\mu$ in (3.24) and $r=\partial q / \partial t$ in (3.25), substract the two relations that we obtain, and find, thanks to (3.2), that

$$
\frac{\alpha}{2} \frac{d}{d t}|\nabla q|^{2}+c\left(\left|\frac{\partial q}{\partial t}\right|^{2}+|\nabla \mu|^{2}\right)+\left(f^{\prime}\left(q_{1}+\varphi\right)-f^{\prime}\left(q_{2}+\varphi\right), \frac{\partial q}{\partial t}\right)=0
$$


We then have, under the assumptions of the theorem,

$$
\left|\left(f^{\prime}\left(q_{1}+\varphi\right)-f^{\prime}\left(q_{2}+\varphi\right), \frac{\partial q}{\partial t}\right)\right|=\left|\int_{\Omega} h\left(q_{1}, q_{2}, t\right) q \frac{\partial q}{\partial t} d x\right|
$$

where

$$
h\left(q_{1}, q_{2}, t\right)=\int_{0}^{1} f^{\prime \prime}\left(s q_{1}+(1-s) q_{2}+\varphi\right) d s
$$

satisfies

$$
\left|h\left(q_{1}, q_{2}, t\right)\right| \leq c\left(\left|q_{1}\right|^{2 p}+\left|q_{2}\right|^{2 p}+c^{\prime}(T)\right) .
$$

We thus find, using Hölder's inequality and the Sobolev embedding theorems, that

$$
\left|\left(f^{\prime}\left(q_{1}+\varphi\right)-f^{\prime}\left(q_{2}+\varphi\right), \frac{\partial q}{\partial t}\right)\right| \leq c(t)|\nabla q|\left|\frac{\partial q}{\partial t}\right|
$$

where $c=c\left(\left\|q_{1}\right\|_{H^{1}(\Omega)},\left\|q_{2}\right\|_{H^{1}(\Omega)}, T\right)$ belongs to $L^{\infty}(0, T)$, so that

$$
\frac{d}{d t}|\nabla q|^{2} \leq c(t)|\nabla q|^{2}
$$

where $c$ belongs to $L^{\infty}(0, T)$, hence the uniqueness for $q$. We then easily obtain the uniqueness for $\mu$.

Remark 3.2. Having the result of Theorem 3.1, we can now interpret (2.12) as a reaction-diffusion equation with right-hand side $\mu-b \cdot \nabla \mu-$ $h \in L^{2}(0, T ; H)$. This yields that $q \in \mathcal{C}([0, T] ; V) \cap L^{2}\left(0, T ; H_{\text {per }}^{2}(\Omega)\right)$ and we can then prove, using Agmon's inequality, the uniqueness for $p \in[1,2]$ if $n=3$ (under the assumptions of Theorem 3.1). Furthermore, if $a=0$ and $q_{0} \in H_{\text {per }}^{2}(\Omega)$, we deduce from (2.11) and from classical regularity results for second-order elliptic systems that $\mu \in L^{2}\left(0, T ; H_{\mathrm{per}}^{2}(\Omega)\right)$ and it then follows from $(2.12)$ that $q \in \mathcal{C}\left([0, T] ; H_{\text {per }}^{2}(\Omega)\right) \cap L^{2}\left(0, T ; H_{\text {per }}^{3}(\Omega)\right)$.

Remark 3.3. If we assume that $m$ is regular enough (say, $m \in L_{\text {loc }}^{\infty}\left(\mathbb{R}^{+}\right.$; $\left.L^{1}(\Omega)\right)$ ), then we deduce from the regularity of $q$ similar regularity results on $\rho$. 
We assume from now on that $B$ is symmetric (and positive definite) and consider (2.16), (2.17), and (2.18). We set $H=L^{2}(\Omega)$ and $V=H_{\text {per }}^{2}(\Omega)$ and we denote by $N$ the operator $-\operatorname{div} B \nabla$ with domain $\dot{H}_{\text {per }}^{2}(\Omega)=\{r \in$ $\left.H_{\mathrm{per}}^{2}(\Omega), \int_{\Omega} r d x=0\right\}$.

We associate with (2.16), (2.17), and (2.18) the following variational formulation, for $T>0$ given.

Find $q:[0, T] \rightarrow V$ such that

$$
\begin{gathered}
\frac{d}{d t}[(q, r)+(q, d \cdot \nabla r)+(\tilde{B} \nabla q, \nabla r)]+\alpha\left(\nabla B^{1 / 2} \nabla q, \nabla B^{1 / 2} \nabla r\right) \\
+\left(B \nabla f^{\prime}(q+\varphi), \nabla r\right)=(k, r), \quad \forall r \in V, \\
q(0)=q_{0} .
\end{gathered}
$$

We assume here, for the sake of simplicity, that $\tilde{B}$ is positive definite. Actually, it would be possible to obtain some existence and uniqueness results when $\tilde{B}$ is only positive semidefinite; see [57].

Finally, we make the following assumptions:

$$
\begin{gathered}
f \text { is of class } \mathcal{C}^{2}, \\
c_{1} s^{2 p+2}-c_{2} \leq f(s) \leq c_{3} s^{2 p+2}+c_{4}, \quad \forall s \in \mathbb{R}, c_{1}, c_{3}>0, c_{2}, c_{4} \geq 0, \\
f^{\prime}(s) s \geq c_{5} f(s)-c_{6}, \quad \forall s \in \mathbb{R}, c_{5}>0, c_{6} \geq 0, \\
\left|f^{\prime}(s)\right| \leq c_{7}|s|^{2 p+1}+c_{8}, \quad \forall s \in \mathbb{R}, c_{7}, c_{8} \geq 0, \\
f^{\prime \prime}(s) \geq-c_{9}, \quad \forall s \in \mathbb{R}, c_{9} \geq 0,
\end{gathered}
$$

where $p \geq 1$ if $n=2$ and $p \in[1,2]$ if $n=3$ (again, polynomials of degree $2 p+2$ with strictly positive leading coefficient satisfy these assumptions);

$$
\begin{gathered}
k \in L_{\mathrm{loc}}^{\infty}\left(\mathbb{R}^{+} ; L^{\infty}(\Omega)\right), \\
\varphi \in \mathcal{C}^{1}\left(\mathbb{R}^{+}\right) .
\end{gathered}
$$

Our aim is now to derive a priori estimates. First, we multiply (2.16) by $r=N^{-1} \bar{q}, \bar{q}=q-(1 / \operatorname{Vol}(\Omega)) \int_{\Omega} q d x$ and integrate over $\Omega$ to obtain, setting $\|\cdot\|_{-1}=\left|N^{-1} \cdot\right|$,

$$
\begin{aligned}
\frac{d}{d t}\left(\|\bar{q}\|_{-1}^{2}\right. & \left.+\left|\tilde{B}^{1 / 2} \nabla B^{1 / 2} \nabla N^{-1} \bar{q}\right|^{2}\right)+c\|q\|_{H^{1}(\Omega)}^{2} \\
& +2 \int_{\Omega} f^{\prime}(q+\varphi) \bar{q} d x \leq c^{\prime}|q|^{2}+c^{\prime \prime}\left|\frac{\partial q}{\partial t}\right|^{2}+c^{\prime \prime \prime}(T) .
\end{aligned}
$$


We have, thanks to (3.36) and (3.37),

$$
\begin{aligned}
& \int_{\Omega} f^{\prime}(q+\varphi) \bar{q} d x \\
& \quad \geq c_{5} \int_{\Omega} f(q+\varphi) d x-c\left(q_{0}, T\right) \int_{\Omega}|q+\varphi|^{2 p+1} d x-c^{\prime}\left(q_{0}, T\right) \\
& \quad \geq c \int_{\Omega} f(q+\varphi) d x-c^{\prime}\left(q_{0}, T\right)
\end{aligned}
$$

which yields

$$
\begin{aligned}
\frac{d}{d t}\left(\|\bar{q}\|_{-1}^{2}\right. & \left.+\left|\tilde{B}^{1 / 2} \nabla B^{1 / 2} \nabla N^{-1} \bar{q}\right|^{2}\right)+c\|q\|_{H^{1}(\Omega)}^{2} \\
& +c^{\prime} \int_{\Omega} f(q+\varphi) d x \leq c^{\prime \prime}|q|^{2}+c^{\prime \prime \prime}\left|\frac{\partial q}{\partial t}\right|^{2}+c^{i v}\left(q_{0}, T\right) .
\end{aligned}
$$

Proceeding similarly for $r=N^{-1}(\partial q / \partial t)$, we find

$$
\begin{aligned}
& \frac{d}{d t}\left(\alpha|\nabla q|^{2}+2 \int_{\Omega} f(q+\varphi) d x\right)+c\left\|\frac{\partial q}{\partial t}\right\|_{-1}^{2} \\
& \quad+c^{\prime}\left|\tilde{B}^{1 / 2} \nabla B^{1 / 2} \nabla N^{-1} \frac{\partial q}{\partial t}\right|^{2} \leq c^{\prime \prime} \int_{\Omega} f(q+\varphi) d x+c^{\prime \prime \prime}(T) .
\end{aligned}
$$

Multiplying finally (2.16) by $q$, we have, integrating over $\Omega$ and using (3.39),

$$
\begin{aligned}
& \frac{d}{d t}\left(|q|^{2}+\mid \tilde{B}^{1 / 2}\right.\left.\left.\nabla q\right|^{2}\right)+c\left|\nabla B^{1 / 2} \nabla q\right|^{2} \\
& \leq c^{\prime}\|q\|_{H^{1}(\Omega)}^{2}+c^{\prime \prime}\left|\frac{\partial q}{\partial t}\right|^{2}+c^{\prime \prime \prime}(T)
\end{aligned}
$$

Combining (3.43), (3.44), and (3.45), we obtain the following theorem.

Theorem 3.4. Assume that (3.35), (3.36), (3.37), (3.38), (3.39), and (3.40) hold and that $q_{0} \in H_{\mathrm{per}}^{1}(\Omega)$. Then, (3.33) and (3.34) possesses at least one solution $q$ such that $q \in \mathcal{C}([0, T] ; H) \cap L^{\infty}\left(0, T ; H_{\text {per }}^{1}(\Omega)\right) \cap L^{2}(0, T ; V)$ and $\partial q / \partial t \in$ $L^{2}(0, T ; H)$, for all $T>0$.

Remark 3.5. We can obtain more regularity by multiplying (2.16) by $\partial q / \partial t$. Furthermore, if we make some growth restrictions on $f^{\prime \prime}$, we can prove the uniqueness of solutions. 
Finally, we consider formulation (2.24), (2.25), and (2.26) (with $\theta$ replaced by $\Phi_{0}$ in (2.26)), with which we associate the variational formulation, for $T>0$ given.

Find $\Phi:[0, T] \rightarrow\left\{v \in H_{\text {per }}^{2}(\Omega), \operatorname{div}(B \nabla v) \in L^{2 p+2}(\Omega)\right\}$ such that

$$
\begin{gathered}
\frac{d}{d t}[(\Phi, r)+(\Phi, d \cdot \nabla r)+(\tilde{B} \nabla \Phi, \nabla r)]+\alpha\left(\nabla B^{1 / 2} \nabla \Phi, \nabla B^{1 / 2} \nabla r\right) \\
-\left(g^{\prime}(\operatorname{div}(B \nabla \Phi)), r\right)=0, \quad \forall r \in H_{\mathrm{per}}^{2}(\Omega) \\
\Phi(0)=\Phi_{0} .
\end{gathered}
$$

We assume here that $\tilde{B}$ is positive definite and that $g$ satisfies (3.3), (3.4), and (3.5).

Multiplying (2.24) by $\Phi, \partial \Phi / \partial t$, and $\operatorname{div}(B \nabla(\partial \Phi / \partial t))$, we obtain, integrating over $\Omega$,

$$
\begin{gathered}
\frac{d}{d t}\left(|\Phi|^{2}+(\tilde{B} \nabla \Phi, \nabla \Phi)\right)+c\|\Phi\|_{H^{2}(\Omega)}^{2} \\
\leq c^{\prime}\left(\int_{\Omega}|\operatorname{div}(B \nabla \Phi)|^{2 p+2} d x\right)^{(2 p+1) /(p+1)}\|\Phi\|_{H^{2}(\Omega)}^{2} \\
+c^{\prime \prime}\|\Phi\|_{H^{1}(\Omega)}^{2}+c^{\prime \prime \prime}+\delta\left|\nabla \frac{\partial \Phi}{\partial t}\right|^{2}, \quad \forall \delta>0, \\
\frac{d}{d t}\left|\nabla B^{1 / 2} \nabla \Phi\right|^{2}+c|| \frac{\partial \Phi}{\partial t} \|_{H^{1}(\Omega)}^{2} \leq c^{\prime}\left(\int_{\Omega}|\operatorname{div}(B \nabla \Phi)|^{2 p+2} d x\right)^{(2 p+1) /(p+1)}+c^{\prime \prime}, \\
\frac{d}{d t}\left(\alpha|\nabla \operatorname{div}(B \nabla \Phi)|^{2}+2 \int_{\Omega} g(\operatorname{div}(B \nabla \Phi)) d x\right)+2\left|B^{1 / 2} \nabla \frac{\partial \Phi}{\partial t}\right|^{2} \\
\quad+2\left(\tilde{B} \nabla B^{1 / 2} \nabla \frac{\partial \Phi}{\partial t}, \nabla B^{1 / 2} \nabla \frac{\partial \Phi}{\partial t}\right)=0 .
\end{gathered}
$$

This yields the following theorem.

Theorem 3.6. Assume that $g$ satisfies (3.3), (3.4), and (3.5) and that $\Phi_{0}$ belongs to $H_{\text {per }}^{3}(\Omega)$. Then, (3.46) possesses at least one solution $\Phi$ such that $\Phi \in \mathcal{C}\left([0, T] ; H^{3-\epsilon}(\Omega)\right) \cap L^{\infty}\left(0, T ; H_{\mathrm{per}}^{3}(\Omega)\right)$ and $\partial \Phi / \partial t \in L^{2}\left(0, T ; H_{\mathrm{per}}^{2}(\Omega)\right)$ for all $\epsilon>0$ and for all $T>0$.

Remark 3.7. Again, we can prove the uniqueness of solutions if $g$ belongs to $C^{2}(R)$ and $g^{\prime \prime}$ satisfies some growth restrictions; in that case, we can also obtain more regularity on the solutions. Finally, we can also prove the existence of solutions (but not the uniqueness) if $\tilde{B}$ is only positive semidefinite. All this will be studied elsewhere. 


\section{References}

[1] H. W. Alt and I. Pawłow, Existence of solutions for non-isothermal phase separation, Adv. Math. Sci. Appl. 1 (1992), no. 2, 319-409.

[2] _ A mathematical model of dynamics of nonisothermal phase separation, Phys. D 59 (1992), no. 4, 389-416.

[3] J. W. Barrett and J. F. Blowey, An error bound for the finite element approximation of the Cahn-Hilliard equation with logarithmic free energy, Numer. Math. 72 (1995), no. 1, 1-20.

[4] - Finite element approximation of the Cahn-Hilliard equation with concentration dependent mobility, Math. Comp. 68 (1999), no. 226, 487-517.

[5] J. W. Barrett, J. F. Blowey, and H. Garcke, Finite element approximation of the Cahn-Hilliard equation with degenerate mobility, SIAM J. Numer. Anal. 37 (1999), no. 1, 286-318.

[6] L. Bertini, C. Landim, and S. Olla, Derivation of Cahn-Hilliard equations from Ginzburg-Landau models, J. Statist. Phys. 88 (1997), no. 1-2, 365-381.

[7] E. Bonetti, P. Colli, W. Dreyer, G. Gilardi, G. Schimperna, and J. Sprekels, On a model for phase separation in binary alloys driven by mechanical effects, Phys. D 165 (2002), no. 1-2, 48-65.

[8] E. Bonetti, W. Dreyer, and G. Schimperna, Global solutions to a generalized Cahn-Hilliard equation with viscosity, Adv. Differential Equations 8 (2003), no. 2, 231-256.

[9] A. Bonfoh, Comportement asymptotique de modèles en transitions de phases, Ph.D. thesis, Université de Poitiers, France, 2001.

[10] A. Bonfoh and A. Miranville, On Cahn-Hilliard-Gurtin equations, Nonlinear Anal. 47 (2001), no. 5, 3455-3466.

[11] F. Boyer, Ecoulements diphasiques de type Cahn-Hilliard, Ph.D. thesis, Université Bordeaux-I, France, 2001.

[12] A theoretical and numerical model for the study of incompressible mixture flows, Computers and Fluids 31 (2002), no. 1, 41-68.

[13] L. A. Caffarelli and N. E. Muler, An $L^{\infty}$ bound for solutions of the Cahn-Hilliard equation, Arch. Rational Mech. Anal. 133 (1995), no. 2, 129-144.

[14] G. Caginalp, The dynamics of a conserved phase field system: Stefan-like, HeleShaw, and Cahn-Hilliard models as asymptotic limits, IMA J. Appl. Math. 44 (1990), no. 1, 77-94.

[15] J. W. Cahn, On spinodal decomposition, Acta Metall. 9 (1961), 795-801.

[16] J. W. Cahn and J. E. Hilliard, Free energy of a nonuniform system I. Interfacial free energy, J. Chem. Phys. 2 (1958), 258-267.

[17] J. W. Cahn and A. Novick-Cohen, Evolution equations for phase separation and ordering in binary alloys, J. Statist. Phys. 76 (1994), 877-909.

[18] M. Carrive, A. Miranville, and A. Piétrus, The Cahn-Hilliard equation for deformable elastic continua, Adv. Math. Sci. Appl. 10 (2000), no. 2, 539-569.

[19] L. Cherfils and A. Miranville, Generalized Cahn-Hilliard equations with a logarithmic free energy, Rev. R. Acad. Cienc. Exact. Fís. Nat. (Esp.) 94 (2000), no. $1,19-32$.

[20] J. W. Cholewa and T. Dlotko, Global Attractors in Abstract Parabolic Problems, London Mathematical Society Lecture Note Series, vol. 278, Cambridge University Press, Cambridge, 2000. 
[21] L. Chupin, An existence result for a mixture of non-newtonian fluids with stressdiffusion using the Cahn-Hilliard formulation, preprint, 2002.

[22] M. I. M. Copetti, Numerical analysis of spectral approximations to the CahnHilliard equation, preprint, 1999.

[23] M. I. M. Copetti and C. M. Elliott, Numerical analysis of the Cahn-Hilliard equation with a logarithmic free energy, Numer. Math. 63 (1992), no. 1, 39-65.

[24] G. Da Prato and A. Debussche, Stochastic Cahn-Hilliard equation, Nonlinear Anal. 26 (1996), no. 2, 241-263.

[25] S. R. de Groot and P. Mazur, Nonequilibrium Thermodynamics, Dover Publications, New York, 1984, reprint of the 1962 original.

[26] A. Debussche and L. Dettori, On the Cahn-Hilliard equation with a logarithmic free energy, Nonlinear Anal. 24 (1995), no. 10, 1491-1514.

[27] M. Efendiev, H. Gajewski, and S. Zelik, The finite dimensional attractor for a 4th order system of Cahn-Hilliard type with a supercritical nonlinearity, Adv. Differential Equations 7 (2002), no. 9, 1073-1100.

[28] M. Efendiev and A. Miranville, New models of Cahn-Hilliard-Gurtin equations, Université de Poitiers, preprint, 2002.

[29] N. Elezović and A. Mikelić, On the stochastic Cahn-Hilliard equation, Nonlinear Anal. 16 (1991), no. 12, 1169-1200.

[30] C. M. Elliott, The Cahn-Hilliard model for the kinetics of phase separation, Mathematical Models for Phase Change Problems (Óbidos, 1988) (J. F. Rodrigues, ed.), Internat. Ser. Numer. Math., vol. 88, Birkhäuser, Basel, 1989, pp. 35-73.

[31] C. M. Elliott and D. A. French, Numerical studies of the Cahn-Hilliard equation for phase separation, IMA J. Appl. Math. 38 (1987), no. 2, 97-128.

[32] _ A nonconforming finite-element method for the two-dimensional CahnHilliard equation, SIAM J. Numer. Anal. 26 (1989), no. 4, 884-903.

[33] C. M. Elliott and H. Garcke, On the Cahn-Hilliard equation with degenerate mobility, SIAM J. Math. Anal. 27 (1996), no. 2, 404-423.

[34] C. M. Elliott and S. Luckhaus, A generalized diffusion equation for phase separation of a multi-component mixture with interfacial energy, preprint, 1991.

[35] C. M. Elliott and Z. Songmu, On the Cahn-Hilliard equation, Arch. Rational Mech. Anal. 96 (1986), no. 4, 339-357.

[36] D. J. Eyre, Systems of Cahn-Hilliard equations, SIAM J. Appl. Math. 53 (1993), no. $6,1686-1712$.

[37] U Unconditionally gradient stable time marching the Cahn-Hilliard equation, Computational and Mathematical Models of Microstructural Evolution (San Francisco, Calif, 1998) (J. W. Bullard, R. Kalia, M. Stoneham, and L. Q. Chen, eds.), Mater. Res. Soc. Sympos. Proc., vol. 529, MRS, Pennsylvania, 1998, pp. 39-46.

[38] X. Feng and A. Prohl, Numerical analysis of the Cahn-Hilliard equation and approximation for the Hele-Shaw problem, Part I: error analysis under minimum regularities, preprint, 2001.

[39] E. Fried and M. E. Gurtin, Continuum theory of thermally induced phase transitions based on an order parameter, Phys. D 68 (1993), no. 3-4, 326-343.

[40] _ Dynamic solid-solid transitions with phase characterized by an order parameter, Phys. D 72 (1994), no. 4, 287-308.

[41] H. Gajewski, On a nonlocal model of non-isothermal phase separation, to appear in Adv. Math. Sci. Appl. 
[42] H. Gajewski and K. Zacharias, On a nonlocal phase separation model, preprint, 2001.

[43] W. J. Gao and J. X. Yin, Systems of Cahn-Hilliard equations with density-dependent transition matrices, Chinese Ann. Math. Ser. A 20 (1999), no. 2, 169176.

[44] H. Garcke, On mathematical models for phase separation in elastically stressed solids, Habilitation thesis, University of Bonn, 2000.

[45] G. Giacomin and J. L. Lebowitz, Phase segregation dynamics in particle systems with long range interactions. I. Macroscopic limits, J. Statist. Phys. 87 (1997), no. 1-2, 37-61.

[46] - Phase segregation dynamics in particle systems with long range interactions. II. Interface motion, SIAM J. Appl. Math. 58 (1998), no. 6, 1707-1729.

[47] M. E. Gurtin, Generalized Ginzburg-Landau and Cahn-Hilliard equations based on a microforce balance, Phys. D 92 (1996), no. 3-4, 178-192.

[48] M. E. Gurtin, D. Polignone, and J. Viñals, Two-phase binary fluids and immiscible fluids described by an order parameter, Math. Models Methods Appl. Sci. 6 (1996), no. 6, 815-831.

[49] G. R. Ierley and S. Shkoller, Smoothing the wrinkles: a new model for surface tension, preprint, 2002.

[50] D. Jacqmin, Calculation of two-phase Navier-Stokes flows using phase-field modeling, J. Comput. Phys. 155 (1999), no. 1, 96-127.

[51] D. Li and C. Zhong, Global attractor for the Cahn-Hilliard system with fast growing nonlinearity, J. Differential Equations 149 (1998), no. 2, 191-210.

[52] C. Liu and S. Shkoller, Sharp interface limits and global existence for the phasefield Navier-Stokes equations, preprint, 2001.

[53] J. Lowengrub and L. Truskinovsky, Quasi-incompressible Cahn-Hilliard fluids and topological transitions, R. Soc. Lond. Proc. Ser. A Math. Phys. Eng. Sci. 454 (1998), no. 1978, 2617-2654.

[54] S. Maier-Paape and T. Wanner, Spinodal decomposition for the Cahn-Hilliard equation in higher dimensions. I. Probability and wavelength estimate, Comm. Math. Phys. 195 (1998), no. 2, 435-464.

[55] Spinodal decomposition for the Cahn-Hilliard equation in higher dimensions: nonlinear dynamics, Arch. Ration. Mech. Anal. 151 (2000), no. 3, 187219.

[56] A. Miranville, Existence of solutions for a Cahn-Hilliard-Gurtin model, C. R. Acad. Sci. Paris Sér. I Math. 331 (2000), no. 10, 845-850.

[57] - Some generalizations of the Cahn-Hilliard equation, Asymptot. Anal. 22 (2000), no. 3-4, 235-259.

[58] Consistent models of Cahn-Hilliard-Gurtin equations with Neumann boundary conditions, Phys. D 158 (2001), no. 1-4, 233-257.

[59] _ Long-time behavior of some models of Cahn-Hilliard equations in deformable continua, Nonlinear Anal. Real World Appl. 2 (2001), no. 3, 273304.

[60] A. Miranville, A. Piétrus, and J. M. Rakotoson, Dynamical aspect of a generalized Cahn-Hilliard equation based on a microforce balance, Asymptot. Anal. 16 (1998), no. 3-4, 315-345. 
[61] B. Nicolaenko and B. Scheurer, Low-dimensional behavior of the pattern formation Cahn-Hilliard equation, Trends in the Theory and Practice of Nonlinear Analysis (Arlington, Tex., 1984) (V. Lakshmikantham, ed.), NorthHolland Math. Stud., vol. 110, North-Holland Publishing, Amsterdam, 1985, pp. 323-336.

[62] B. Nicolaenko, B. Scheurer, and R. Temam, Some global dynamical properties of a class of pattern formation equations, Comm. Partial Differential Equations 14 (1989), no. 2, 245-297.

[63] A. Novick-Cohen, Energy methods for the Cahn-Hilliard equation, Quart. Appl. Math. 46 (1988), no. 4, 681-690.

[64] - On the viscous Cahn-Hilliard equation, Material Instabilities in Continuum Mechanics (Edinburgh, 1985-1986) (J. M. Ball, ed.), Oxford Sci. Publ., Oxford University Press, New York, 1988, pp. 329-342.

[65] - The Cahn-Hilliard equation: mathematical and modeling perspectives, Adv. Math. Sci. Appl. 8 (1998), no. 2, 965-985.

[66] , Triple-junction motion for an Allen-Cahn/Cahn-Hilliard system, Phys. D 137 (2000), no. 1-2, 1-24.

[67] W. X. Shen and S. M. Zheng, On the coupled Cahn-Hilliard equations, Comm. Partial Differential Equations 18 (1993), no. 3-4, 701-727.

[68] R. Temam, Infinite-Dimensional Dynamical Systems in Mechanics and Physics, 2nd ed., Applied Mathematical Sciences, vol. 68, Springer-Verlag, New York, 1997.

Alain Miranville: Laboratoire d'Applications des Mathématiques, Université de Poitiers, SP2MI, Téléport 2, boulevard Marie et Pierre Curie, 86962 Chasseneuil Futuroscope Cedex, France

E-mail address: miranv@mathlabo.univ-poitiers.fr 


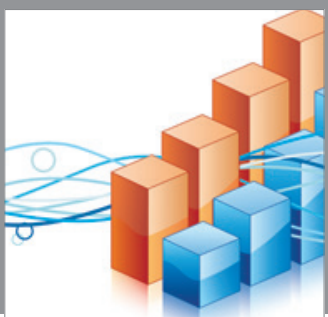

Advances in

Operations Research

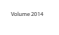

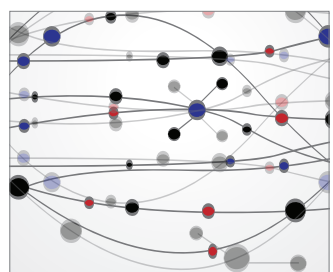

\section{The Scientific} World Journal
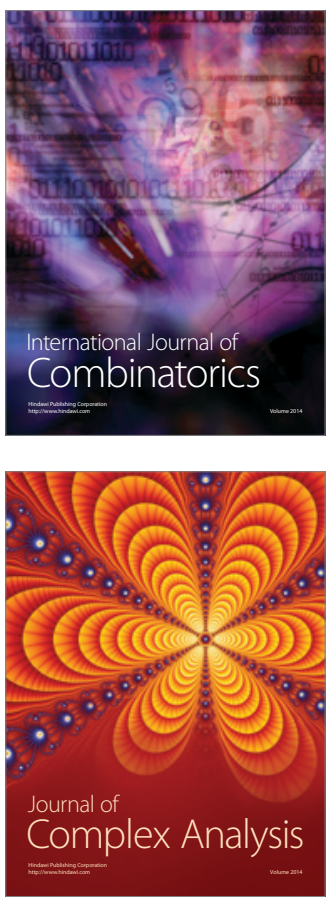

International Journal of

Mathematics and

Mathematical

Sciences
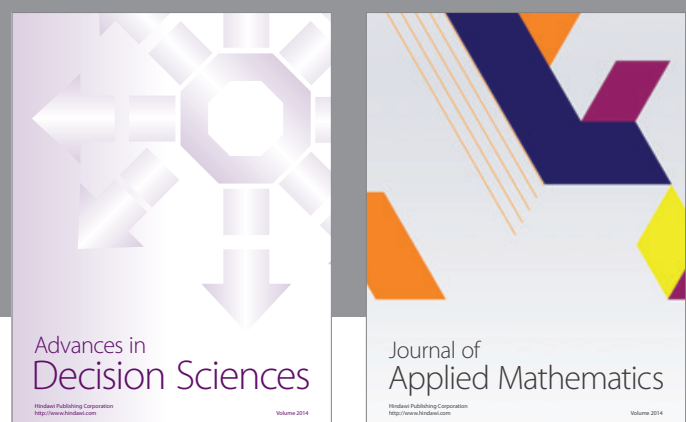

Journal of

Applied Mathematics
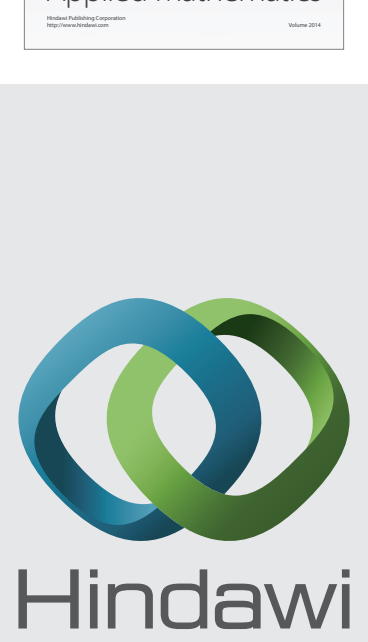

Submit your manuscripts at http://www.hindawi.com
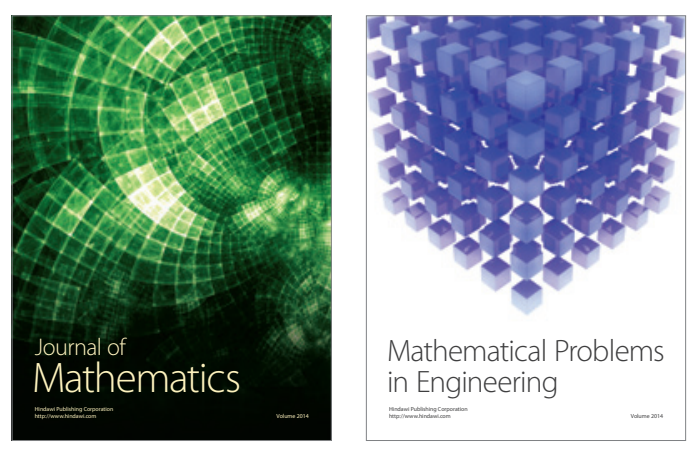

Mathematical Problems in Engineering
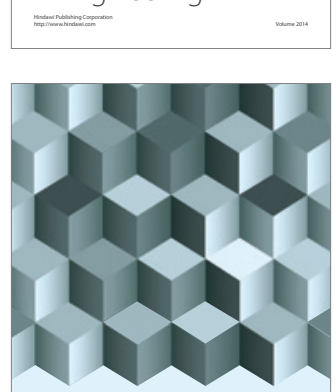

Journal of

Function Spaces
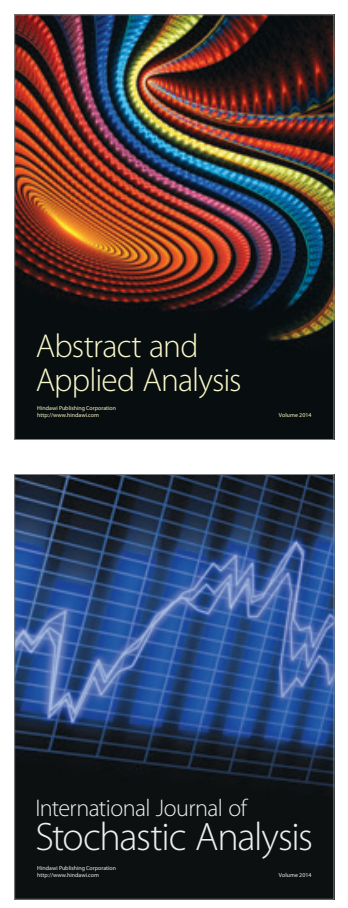

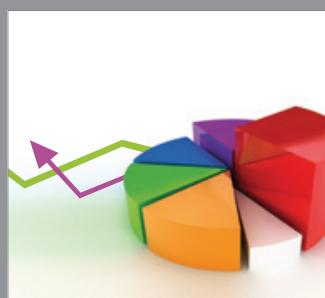

ournal of

Probability and Statistics

Promensencen
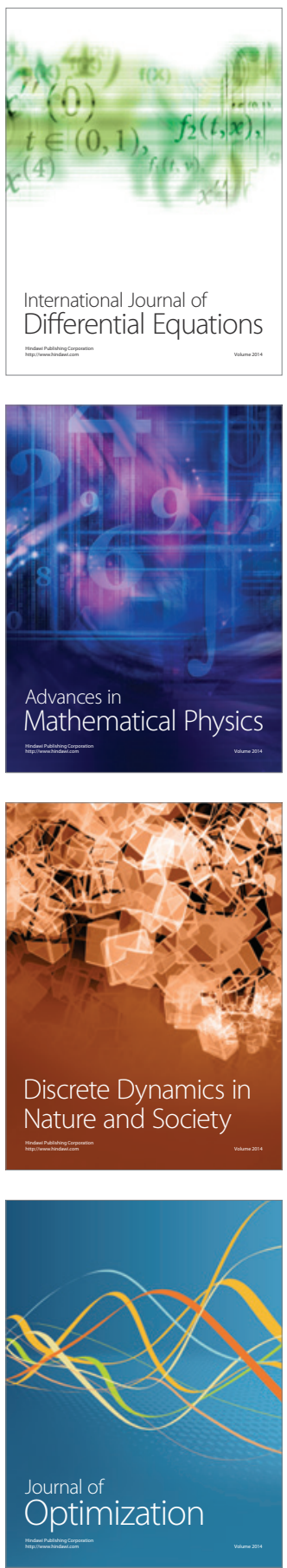